\title{
Rapid carbohydrate utilization test for the identification of Neisseria gonorrhoeae
}

\author{
H. YOUNG, I. C. PATERSON, AND D. R. MCDONALD \\ Department of Bacteriology, University Medical School, Edinburgh, EH8 9 AG
}

\section{Summary}

A rapid carbohydrate utilization test for the identification of $N$. gonorrhoeae was investigated, with reference to its use in a routine diagnostic laboratory. The rapid test was shown to give accurate results in agreement with those of a conventional serum-free sugar medium. Because of the shorter time taken for the confirmation of an isolate, and several other advantages, it is proposed that the rapid test is an extremely useful alternative to conventional sugar tests.

Immunofluorescence was also used to identify isolates of $N$. gonorrhoeae and the rapid carbohydrate utilization test was found to assist in differentiating between $N$. gonorrhoeae and $N$. meningitidis when equivocal or negative immunofluorescence results were obtained.

\section{Introduction}

Although a presumptive diagnosis of gonococcal infection may be made by examination of Gramstained smears whilst the patient is at the clinic, this must be supplemented and confirmed by culture if the maximum number of positive results is to be obtained (Jephcott, Morton, and Turner, 1974). Confirmation of the diagnosis by the isolation of oxidase-positive, Gram-negative diplococci and biochemical proof of identity, normally takes 3 to 4 days and the delay is sometimes due to the failure to demonstrate acid production from glucose only.

A more rapid confirmatory diagnosis followed by immediate treatment would be helpful in the control of the disease. Consequently, immunofluorescence techniques (Hare, 1974) and a rapid fermentation test (RFT) (Kellogg and Turner, 1973) have been investigated.

Kellogg and Turner (1973) reported that the RFT was suitable for the confirmation of identity of Neisseria gonorrhoeae from either primary isolation

Received for publication June 30, 1975

Address for reprints: Dr. H. Young, as above media or purification media. In the RFT, pre-formed enzyme is measured by adding a suspension of the overnight growth of the suspect organism to a buffered (non-nutrient) solution containing the sugar to be tested and a $\mathrm{pH}$ indicator. Since the test measures pre-formed enzyme, it has the advantage of being independent of growth. In contrast, in the conventional technique, a serum-free agar medium containing the appropriate carbohydrate is inoculated with the test organism (Flynn and Waitkins, 1972). A positive reaction is dependent on adequate growth of the test organism and this may take up to $48 \mathrm{hrs}$. In addition, several strains of $N$. gonorrhoeae grow very poorly on the test medium containing maltose, making it impossible to score a valid negative result in the absence of adequate growth.

Unfortunately, as reported by Brown (1974), several laboratories were unable to obtain results comparable to those described by Kellogg and Turner (1973). In view of these conflicting reports, we decided to investigate a rapid carbohydrate utilization test (RCUT), in parallel with the conventional sugar technique and immunofluorescence, and to determine its value in a routine diagnostic laboratory.

\section{Material and methods \\ Bacterial strains}

The best conditions for the RCUT were established using stock strains of $N$. gonorrhoeae and $N$. meningitidis. These were $N$. gonorrhoeae strain 9 , a gift from Dr. D. S. Kellogg, Center for Disease Control, Atlanta, Georgia, and $N$.gonorrhoeae strains $1,2,3,6$, and $N$. meningitidis groups A, B, C, D, E, 29E, W-135, X, and Z, originally obtained from the Neisseria Repository, Berkeley, California, and kindly made available by Professor B. P. Marmion of our Department. Cultures of commensal Neisseria species were provided by Dr. Margaret Calder, Bacteriology Laboratory, City Hospital, Edinburgh.

\section{Specimens}

To assess the test in routine use, specimens were obtained from patients attending the Department for Sexually Transmitted Diseases, The Royal Infirmary of Edinburgh. Material for culture, normally from the urethra, cervix, 
and rectum in female cases and from the urethra in male cases, was plated directly on to Columbia agar base (Ellner, Stoessel, Drakeford, and Vasi, 1966) enriched with 10 per cent. heated human blood; 80 per cent. of the concentrations of vancomycin, colistin, and nystatin recommended by Thayer and Martin (1966) were incorporated. The cultures were processed in a different section of the laboratory as part of the normal diagnostic work; this included sugar utilization tests using a serumfree medium (Flynn and Waitkins, 1972). Any isolates of Gram-negative diplococci were then passed to us for investigation by the RCUT and immunofluorescence techniques outlined below.

\section{Rapid carbohydrate utilization test (RCUT)}

Buffer-salt solution $5.2 \mathrm{mM} \quad \mathrm{K}_{2} \mathrm{HPO}_{4} / \mathrm{KH}_{2} \mathrm{PO}_{4}$ buffer containing 0.8 per cent. $\mathrm{KCl}$ and 0.1 per cent. phenol red, $\mathrm{pH} 7 \cdot 1-7 \cdot 15$. This solution, which is a modification of those of Kellogg and Turner (1973) and Brown (1974), was made by mixing the following solutions : $40 \mathrm{ml} .0 \cdot 1$ $\mathrm{M}-\mathrm{K}_{2} \mathrm{HPO}_{4}, 12 \mathrm{ml}$. 0.1 $\mathrm{M}-\mathrm{KH}_{2} \mathrm{PO}_{4}, 100 \mathrm{ml} .8$ per cent. (w/v) $\mathrm{KCl}, 10 \mathrm{ml} .1$ per cent. (w/v) aqueous phenol red, and $838 \mathrm{ml}$. sterile distilled water. The solution was dispensed in $20-\mathrm{ml}$. amounts in Universal bottles and stored at $-20^{\circ} \mathrm{C}$.

Sugar solutions $100 \mathrm{ml}$. of 10 per cent. (w/v) stock solutions of glucose, maltose, sucrose, fructose, and lactose were prepared using sterile distilled water and stored at $-20^{\circ} \mathrm{C}$. in 4-ml. amounts. Several batches of maltose contained excessive amounts of glucose which produced false positive results. Extra pure maltose (BDH Chemicals Ltd., Poole, England) gave satisfactory results and was used throughout this study. Each week one bottle of buffer-salt solution and one bottle of each sugar was thawed and stored at $4^{\circ} \mathrm{C}$.

Cultures Presumptive positive growth from the primary isolation plate was inoculated on to Difco GC base supplemented with L-glutamine, thiamine pyrophosphate, ferric nitrate, and glucose as described by Kellogg, Peacock, Deacon, Brown, and Pirkle (1963), except that the glucose concentration was reduced from 0.4 to 0.2 per cent. Plates were incubated overnight at $37^{\circ} \mathrm{C}$. in an atmosphere containing 10 per cent. $\mathrm{CO}_{2}$. After overnight incubation, each culture was checked for purity by Gram-staining and the oxidase reaction was performed by flooding a small area of the surface of the plate with a 1.0 per cent. ( $w / v)$ solution of tetramethyl-p-phenylenediamine dihydrochloride.

In addition, several of the stock strains of pathogenic Neisseriae were inoculated on to Thayer-Martin medium and incubated as above before the RCUT was performed on the overnight growth.

Setting up the RCUT Four tubes $(70 \times 10 \mathrm{~mm}$.) are required for each culture to be tested. Using a Finn pipette ${ }^{\star} 20 \mu l .10$ per cent. (w/v) glucose, maltose, and sucrose were added to individual tubes followed by $100 \mu$ l. buffer-salt solution. A thick suspension of overnight growth was made in $300 \mu$. of the buffer-salt solution, mixed well with a Pasteur pipette, and $30 \mu l$.

^Buckley Membranes, 24 Clifton Road, Amersham, Buckinghamshire were transferred to each sugar-containing tube. The tubes were then shaken and incubated at $37^{\circ} \mathrm{C}$. in a waterbath. Cultures giving weak or no fluorescence on the primary isolation plate were tested against fructose and lactose in addition to glucose, maltose, and sucrose. Normally a reading could be made after 30 to $60 \mathrm{~min}$., but incubation was continued for $3 \mathrm{hrs}$ before a definitive reading was made:

Positive-yellow (occasionally, yellow-orange)

Negative-red.

Immunofluorescence At the time of inoculating the plate for the RCUT, an extremely light suspension of the suspect colony from the primary isolation plate was made by emulsifying part of the colony in $200 \mu \mathrm{l}$. distilled water; $10 \mu \mathrm{l}$. of the suspension were immediately transferred to a spot on a 12-well multi-spot slide ${ }^{\star}$ and allowed to dry in air. Stock cultures of $N$. gonorrhoeae and $N$. meningitidis were also tested on each slide. Fluorescein-labelled antigonococcal conjugate $\dagger 10 \mu \mathrm{l}$. was added to each spot and the slide incubated in a moist chamber at $37^{\circ} \mathrm{C}$. for $10 \mathrm{~min}$. The slide was then washed in phosphate-buffered saline (Bacto FA, Difco) and distilled water; $5 \mathrm{~min}$ in each. The dried slides were mounted in buffered glycerol (FA mounting fluid, Difco) and examined under ultra-violet light using a Zeiss RA microscope and a $\times 100$ objective.

\section{Results}

All stock strains of pathogenic Neisseriae and clinical isolates of commensal Neisseria species gave the correct carbohydrate utilization pattern with the RCUT. The results for the commensal Neisseria species were also confirmed using conventional serum-free sugar medium.

Table I shows the source of the 97 isolates from 48 male and 32 female patients, the results obtained by the RCUT and immunofluorescence, and the identity of each isolate as determined by conventional techniques.

Ninety of the isolates were identified as $N$. gonorrhoeae and seven as $N$. meningitidis. The RCUT gave the correct carbohydrate utilization pattern in each case. Immunofluorescence results also showed high agreement with both the RCUT and conventional techniques. Only four of the ninety strains subsequently identified as $N$. gonorrhoeae gave a doubtful reaction on first examination; one of these was a 24-hr culture which, even after subculture, always gave a doubtful or very weak fluorescence, while the remaining three were $72-\mathrm{hr}$ cultures, all of which gave good fluorescence when $24-\mathrm{hr}$ subcultures were tested.

A direct comparison of the time taken for complete identification by immunofluorescence, the RCUT, and conventional methods is shown in Table II.

All the isolates from the 28 cases shown in Table II were $N$. gonorrhoeae, $60 \cdot 7$ per cent. of which were

^C. A. Hendley \& Co., Victoria Road, Buckhurst Hill, Essex †Difco Laboratories, U.K. Division, P.O. Box 14B, Central Avenue, East Molesley, Surrey 
TABLE I Results of immunofluorescence and rapid carbohydrate utilization tests on 97 isolates

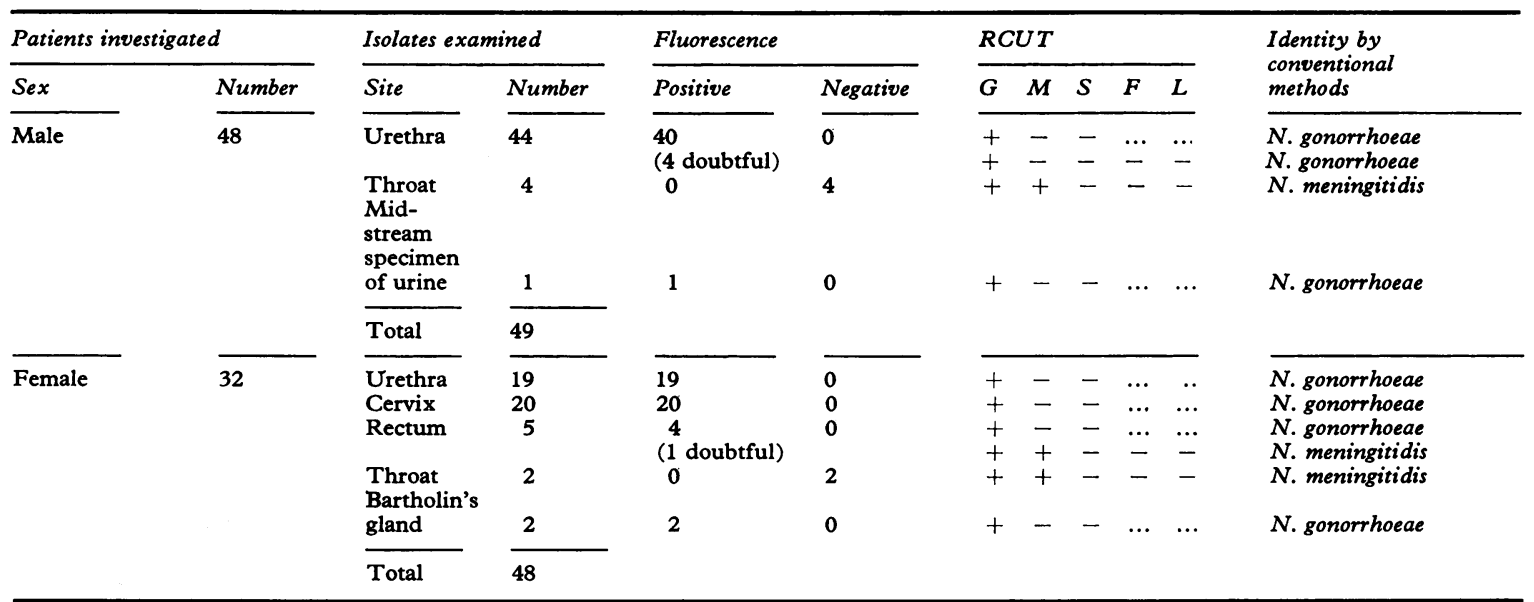

$\mathrm{G}=$ glucose, $M=$ maltose, $\mathrm{S}=$ sucrose, $\mathrm{F}=$ fructose, $\mathrm{L}=$ lactose.

$+=$ acid production $\quad-=$ no acid production

TABLE II Comparison of time taken for complete identification by immunofluorescence and/or rapid carbohydrate utilization and conventional methods in 28 cases

\begin{tabular}{|c|c|c|c|c|c|c|}
\hline \multirow{3}{*}{$\begin{array}{l}\text { Time } \\
\text { (hrs) }\end{array}$} & \multicolumn{6}{|c|}{ Number of cases identified by } \\
\hline & \multicolumn{2}{|c|}{ Immunofluorescence } & \multicolumn{2}{|c|}{$R C U T$} & \multicolumn{2}{|c|}{$\begin{array}{l}\text { Conventional } \\
\text { techniques }\end{array}$} \\
\hline & No. & Per cent. & No. & Per cent. & No. & Per cent. \\
\hline 24 & 17 & $60 \cdot 7$ & 0 & - & 0 & - \\
\hline 48 & 11 & $39 \cdot 3$ & 16 & $57 \cdot 2$ & 11 & $39 \cdot 3$ \\
\hline 72 & 0 & - & 12 & $42 \cdot 8$ & 11 & $39 \cdot 3$ \\
\hline 96 & 0 & - & 0 & - & 6 & $21 \cdot 4$ \\
\hline
\end{tabular}

identified at $24 \mathrm{hrs}$ using immunofluorescence; the remaining 39.3 per cent. were identified at $48 \mathrm{hrs}$. In 57.2 per cent. of the total cases, or sixteen of the seventeen cases positive at $24 \mathrm{hrs}$, an RCUT result at $48 \mathrm{hrs}$ confirmed the fluorescence result. One subculture was contaminated with Gram-positive cocci and had to be freshly subcultured before the RCUT was carried out at $72 \mathrm{hrs}$. By $72 \mathrm{hrs}$ all the isolates had been identified by a combination of immunofluorescence and the RCUT compared with 78.6 per cent. identified at $72 \mathrm{hrs}$ by conventional techniques.

\section{Discussion}

Our investigation confirms that a rapid carbohydrate utilization technique for the identification of $N$. gonorrhoeae can give reliable results in a much shorter time than the conventional technique. The majority of strains gave positive reactions in 30 to $60 \mathrm{~min}$., although incubation was always continued for $3 \mathrm{hrs}$ before a definitive reading was made. In the RCUT system described, the danger of early reading is minimized since strains of $N$. meningitidis invariably gave a positive reaction with maltose quicker than with glucose.

In pilot experiments the buffer solutions described by Kellogg and Turner (1973) and Brown (1974) were found to be unsatisfactory. When we used thick suspensions in order to obtain a rapid result there was a tendency for the maltose tube to give a positive reaction, and when we used a thin suspension changes in the glucose tube were slower and sometimes incomplete. We overcame these problems by increasing the molarity of the buffering system and the proportion of the basic salt. These changes, along with an increase in the amount of phenol red indicator, gave rapid, easy to read, reproducible, results.

When Thayer-Martin medium was used to subculture strains before testing, we found that the growth was difficult to remove from the surface of the medium and did not form a uniform suspension, even after being mixed with a Pasteur pipette. The enzyme activity of the organisms appeared to be lower when they were grown on Thayer-Martin medium, and the colour change was slower and less pronounced than when they were grown on the supplemented Difco GC base. Possibly the glucose in the latter medium has the effect of increasing the carbohydrate metabolizing activity of the cells by enzyme induction. Therefore, in contrast to the findings of Kellogg and Turner (1973), we consider that the amount of growth normally obtained on ThayerMartin primary isolation plates would be unsuitable as a source of material for the RCUT. 
In laboratories where identification of $N$. gonorrhoeae is undertaken by immunofluorescence there is still a need for carbohydrate utilization tests, since there have been reports of cross-reaction between $N$. gonorrhoeae and $N$. meningitidis in immunofluorescence techniques (Reyn, 1969; Hare, 1974). Also, $N$. meningitidis may be implicated in urogenital infection (Beck, Fluker, and Platt, 1974) and the incidence of pharyngeal gonococcal infection continues to increase in certain countries (Wallin, 1975), so that it is important for epidemiological and clinical purposes to distinguish these organisms carefully. The application of the RCUT to Gram-negative diplococci giving weak fluorescence or none provides a means of detecting gonococci antigenically distinct from those used in the preparation of the antigonococcal conjugate and a safeguard against crossreaction with $N$. meningitidis.

We conclude that the RCUT can speed the confirmation of an isolate as $N$. gonorrhoeae. As pointed out by Kellogg and Turner (1973), the reagents for the test are easily prepared and can be stored for several months at $-20^{\circ} \mathrm{C}$. in contrast to the limited shelf-life of conventional media. The obvious danger inherent in inoculating sugar-test media from a selective medium does not apply to the RCUT. The
RCUT, as described here, is thus a useful alternative to the conventional sugar method.

We should like to acknowledge the co-operation of the staff of the University Bacteriology Laboratory, The Royal Infirmary, Edinburgh.

Thanks are also extended to Dr. R. R. Gillies for valuable advice during the preparation of this paper and to Mrs. Joan Collins for typing the manuscript.

\section{References}

Beck, A., Fluker, J. L., and Platt, D. J. (1974) Brit. f. vener. Dis., 50, 367

Brown, W. J. (1974) Appl. Microbiol., 27, 1027

Ellner, P. D., Stoessel, C. J., Drakeford, E., and VAsI, F. (1966) Amer. F. clin. Path., 45, 502

FlynN, J., and Waitkins, S. A. (1972) f. clin. Path., 25, 525

HARE, M. J. (1974) Brit. F. vener. Dis., 50, 437

Jephcott, A. E., Morton, R. S., and Turner, E. B. (1974) Lancet, 2, 1311

Kellogg, D. S., Peacock, W. L., Deacon, W. E., Brown, L., and PirkLE, C. I. (1963) F. Bact., 85, 1274 and Turner, E. M. (1973) Appl. Microbiol., 25, 550

ReYN, A. (1969) Bull. Wld Hlth Org., 40, 245

Thayer, J. D., and Martin, J. E. (1966) Publ. Hlth Rep. (Wash.), 81, 559

WALLIN, J. (1975) Brit. F. vener. Dis., 51, 41 